\title{
Leaving The Emergency Department Without Being Seen or Without Complete Treatment; A Study From Saudi Arabia
}

\author{
Mishal Almarshady, Sharafaldeen Bin Nafisah, Yousef Almubarak, and Husam Althobyane
}

\begin{abstract}
Background The attitude of Leaving the emergency department (ED) without being seen or without completing treatment is attributed to prolonged waiting time. The impact of such behaviour and fate of such patients remain undetermined. Aim This study aimed to investigate the prevalence of Left Without Being Seen (LWBS) and Left Without Completing Treatment (LWCT), analyse the contributing factors for such behaviour and the mortality rate within one week of leaving the ED. Methods A retrospective observational study was performed in a tertiary hospital in Riyadh, Saudi Arabia, for three months. All adult patients of more than 14 years of age, who visited the ED and LWBS or LWCT were included. Patients were seen by physicians and triaged-out, and those who were directed towards the outpatient clinic were excluded. Patients were asked about the reason for leaving, and their intention to return to the same ED again via call. Results The total number of $L W B S$ and LWCT was 286 patients, with a response rate of $75 \%$. The mean age was 45.8 years. About $46.6 \%$ sought medical help within seven days of leaving ED. However, the mortality rate was nil. Many patients $(69.3 \%)$ reported their intention to revisit the same ED in the near future. A positive correlation was found between increasing age and admission within the same seven days' period. Conclusion Prolonged waiting time is a public health concern that needs management strategies, the number of patients whom LWBS and LWCT require continuous monitoring and exploration.
\end{abstract}

\section{INTRODUCTION}

The prolonged waiting time in the Emergency Department (ED) emerged as a public health concern. It resulted in the emergence of a subset of patients who Left Without Being Seen (LWBS) and Left Without Completing the Treatment (LWCT). The former designates a patient encounter that ended with leaving the ED before seen and assessed. The latter, however, designates leaving before completing their treatment plan. Nevertheless, such concern exists irrespective of the healthcare system, or settings-such patients' attitudes are seen in Australia [1], Ireland [2], Canada [3], France [4] and the USA [5]. Noticeably, the LWBS percentage varies between countries between $7.5 \%$ and to as less as $1.41 \%$, over an 8 year of observation [2].

The concern arises on whether such patients needed to revisit the $\mathrm{ED}$, or required admission shortly after leaving. Exploring the predictors for admission is essential for safety and quality projects. The period that reflected the patient's

Mishal Almarshady, Sharafaldeen Bin Nafisah, Yousef Almubarak are with Emergency Department, King Fahd Medical City, Riyadh, Saudi Arabia

Husam Althobyane is with Emergency Department, Almadina Almonawara Hospital, Medina, Saudi Arabia, e-mail: dr.althobyane@gmail.com (Corresponding author). fate after absconding ranged from two days, one week to one month. A consistency is noted in the literature for using one-week's duration as a benchmark [1], [4]. Nonetheless, the reported admission rate within one week revealed a percentage that varied between 2\% [4] and 5\% [1]. Moreover, it is prudent to investigate the mortality rate of such patients. The literature revealed a range from $0.17 \%$ [6] to $35 \%$ [7], whereas several other studies lacked such data [1], [2], [8].

Therefore, this study aimed to investigate the prevalence of LWBS and LWCT in the ED of a large tertiary hospital in Saudi Arabia. It also aimed to analyse the contributing factors for such behaviour and the mortality rate within one week of leaving the ED.

\section{METHODS:}

A retrospective observational study was conducted in King Fahad Medical City (KFMC) ED department. The hospital is a tertiary hospital in Riyadh of Saudi Arabia with a median of $120 \pm 20$ patients' visit per day. To better capture such behaviour, the cases were analysed three months before the emergence of COVID-19 pandemic in March 2020. LWBS were defined as those who register in ED but leave without initial assessment. LWCT, on the other hand, defined as patients who are initially assessed by an ED physician but leave the hospital before the completion of their treatment plan. The inclusion criteria were all adult patients of more than 14 years of age who were labelled as LWBS and LWCT. On the other hand, the exclusion criteria encompass those who triaged out and those who were directed towards primary healthcare or employee clinic. After seven days of leaving the ED, patients included in the study were contacted three attempts made to contact each patient, with a one-day interval between each attempt. If the patient was admitted, the inpatient nurse or a family member was asked to approach the patient to answer the inquiry, or if the patient was unable to answer the call, the patient's companion was eligible to provide answers. Demographics information, the patient'sstatus during the seven days and whether he/she required admission were collected. The reason for leaving the ED and the intention to visit the same ED in the near future were inquired.

\section{RESULTS}

The total number of patients who LWBS and LWCT were 286 patients during the three months. We were unable to reach 34 patients because their telephone number was not registered 
correctly. Of the 252 patients, 189 patients agreed to participate in the present survey, which comprised a total followup rate of $75 \%$. The patient's entire triaging category ranged between category two in $0.79 \%(n=2), 92.1 \%(n=232)$ three and $7.14 \%(\mathrm{n}=18)$ for category four based on the Canadian Triage and Acuity Scale (CTAS). Those with category two were LWCT. The first is a thirty-six-year-old lady who was stabilised and did not seek medical help within one week of the event. The second was fifty years old refused to participate in our survey. However, none of those patients had a history of cancer, and at the time of this writing, they are doing well. The demographics were as follow; the mean age was 45.8 years old, ranging between 17 to 87. Other demographics illustrated in Table 1. The number of LWBS and LWCT illustrated in Figure 1.

The mortality rate was nil. Less than half of the patients, $46.6 \%(\mathrm{n}=88)$ sought medical help within seven days. The admission rate within the same period was $7.9 \%(\mathrm{n}=15)$. Despite this, $69.3 \%(n=131)$ reported their intention to revisit the same ED in the near future. The plan to visit the same ED did not differ between those who received initial treatment from those who did not, $p>0.05$. Neither did the age, nor the gender influenced such perception, $\mathrm{p}>0.05$.

The main reason reported by most patients, $85.7 \%(\mathrm{n}=167)$, irrespective of LWBS or LWCT was the long waiting time. Other causes included staff attitude, family related issues, and lack of policy that prioritised oncology patients. Some patients felt assured after their vital signs were obtained, while others reported having a near appointment.

A difference was noted between LWBS and LWCT concerning seeking medical attention within one week of their absconding; Fisher's exact test $=8.53, \mathrm{p}<0.05$. The majority $(86.7 \%)$ of those who received initial treatment were less likely to seek other medical help in the following one week. In contrast, half of the patients whom LWBS sought medical help than those who did not $(50 \%, 49.4 \%$, respectively) illustrated in the Figure 2. However, the admission rate did not differ between the two groups; Fisher's exact test $=5.01, \mathrm{p}>0.05$.

Additionally, neither did the age nor the gender of the patients influenced LWBS or LWCT, $p>0.05$. Nonetheless, the older the patient, the more likely he/she were admitted with a mean of $54, \mathrm{SD}=20.85$ compared to a mean of $44.82, \mathrm{SD}=$ $15.47, \mathrm{t}(17)=1.98, \mathrm{p}<0.05$. Moreover, patients following with oncology service did not differ from other patients concerning their absconding with or without initial treatment $(\mathrm{p}>0.05)$.

This study was carried out over a three-month period which can be seen as a limitation. A prolonged analysis is warranted to capture such behaviour and attitude better.

\section{DISCUSSION}

In the present study, it was observed that LWCT was far less than those who were LWBS. However, almost half of the patients sought medical help within one week. However, this study was unable to find significant distinct contributing factors for LWBS or LWCT. On the other hand, the mortality rate was nil, even for those who did not agree to participate. Negative feelings towards the service might develop in patients leaving the ED before an assessment, which is expected to reflect the questionnaire response rate negatively. The present study's response rate was $75 \%$, which was far from the reported rates from different countries, and hence generalizability might not be erroneous [1], [3], [4], [9], [7], [10], [11]. Interestingly, the patient's reflection on the emergency service did not alter, since two-third reported their willingness to visit the same ED in the near future, irrespective of whether they received initial treatment or not.

The available data uncovered a low admission rate within one week of leaving the ED. However, since increasing age was positively correlated with a high likelihood for admission, calling patients back or arranging a follow-up within the oneweek targets the elderly in particular. It is also prudent to integrate an initial assessment room for initial management, whether as a part of the permanent patient's flow-management or when the waiting time is expected to be prolonged. In the present setting, oncology patients have access to the Oncology Treatment Unit (OUT), representing a safety net for such patients. The authors urge the extrapolation of such outpatient service to those prone for admission after absconding.

\section{CONCLUSION}

Prolonged waiting time remains a public health concern worldwide. It was found that the main reason for leaving ED, whether without being seen or after the initial assessment is prolonging ED time. Strategies targeting prolonged waiting time is imperative while continuing to assess for its benefit and effect, especially on those who left without being seen or left after the initial assessment.

\section{LIST OF ABBREVIATIONS:}

LWBS: Left Without Being Seen

LWCT: Left Without Completing Treatment

\section{REFERENCES}

[1] M. Mohsin, R. Forero, S. Ieraci, A. E. Bauman, L. Young and N. Santiano, "A population follow-up study of patients who left an emergency department without being seen by a medical officer," Emergency Medicine Journal, vol. 24, no. 3, pp. 175179, 2007. [Online]. Available: 10.1136/emj.2006.038679; https: //dx.doi.org/10.1136/emj.2006.038679

[2] P. Gilligan, D. Joseph, S. Winder, F. O. Keeffe, O. Oladipo, T. Ayodele, Q. Asuquo, P. O'Kelly, and D. Hegarty, "DNW-"Did Not Wait" or "Demographic Needing Work": a study of the profile of patients who did not wait to be seen in an Irish emergency department," Emergency Medicine Journal, vol. 26, no. 11, pp. 780782, 2009. [Online]. Available: 10.1136/emj.2008.063388; https: //dx.doi.org/10.1136/emj.2008.063388

[3] J. Fraser, P. Atkinson, A. Gedmintas, M. Howlett, R. McCloskey, and J. French, "A comparative study of patient characteristics, opinions, and outcomes, for patients who leave the emergency department before medical assessment," CJEM, vol. 19, no. 5, pp. 347-354, 2017. [Online]. Available: 10.1017/cem.2016.375; https: //dx.doi.org/10.1017/cem.2016.375

[4] G. Ibanez, L. Guerin, and N. Simon, "Which improvements could prevent the departure of the left-without-being-seen patients?" Emergency Medicine Journal, vol. 28, no. 11, pp. 945-947, 2011. [Online]. Available: 10.1136/emj.2009.086397;https://dx.doi.org/ 10.1136/emj.2009.086397

[5] R. Y. Hsia, S. M. Asch, and R. E. Weiss, "Hospital determinants of emergency department left without being seen rates," Ann Emerg Med, vol. 58, pp. 24-32, 2011. 


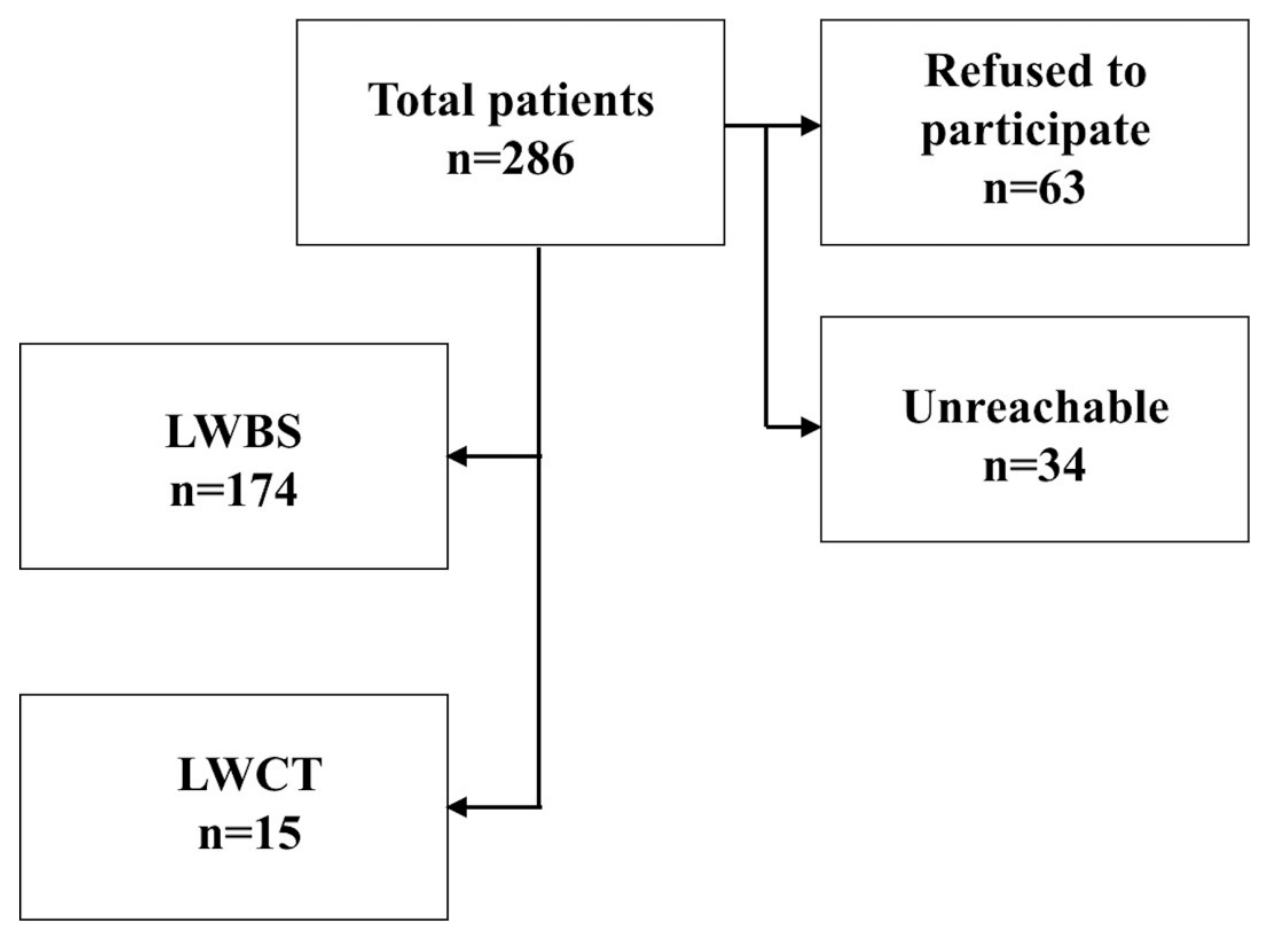

Fig. 1. Illustrates the number of total participants and LWBS and LWCT.

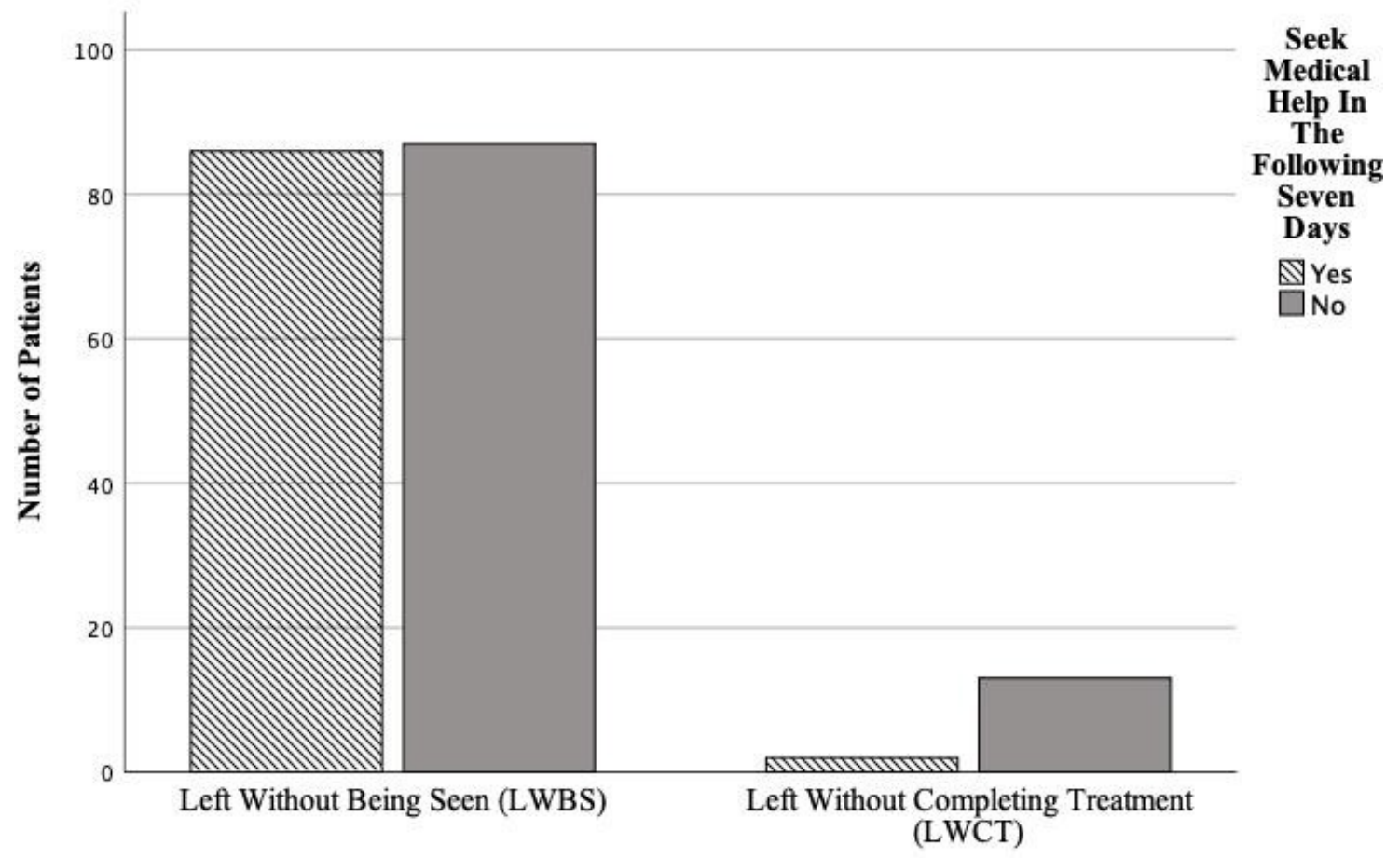

Fig. 2. 2. Illustrates the comparison between those who LWBS and LWCT concerning seeking medical attention within seven days of leaving the ED. 
TABLE I

THE DEMOGRAPHICS OF THE PATIENTS WHO WERE LWBS AND LWCT.

\begin{tabular}{lll}
\hline $\begin{array}{l}\text { Demographics } \\
\text { Gender }\end{array}$ & NUMBER (n) & Percentage (\%) \\
Males & 86 & 45.5 \\
Females & 102 & 54 \\
Oncology patients & 33 & 17.5 \\
\hline
\end{tabular}

[6] J. Tropea, V. Sundararajan, A. Gorelik, M. Kennedy, P. Cameron, and C. A. Brand, "Patients Who Leave Without Being Seen in Emergency Departments: An Analysis of Predictive Factors and Outcomes," Academic Emergency Medicine, vol. 19, no. 4, pp. 439-447, 2012. [Online]. Available: 10.1111/j.1553-2712.2012.01327.x;https: //dx.doi.org/10.1111/j.1553-2712.2012.01327.x

[7] C. M. Fernandes, M. R. Daya, S. Barry, and N. Palmer, "Emergency department patients who leave without seeing a physician: The Toronto Hospital experience," Annals of Emergency Medicine, vol. 24, no. 6, pp. 1092-1096, 1994. [Online]. Available: 10.1016/s0196-0644(94)702381;https://dx.doi.org/10.1016/s0196-0644(94)70238-1

[8] K. P. Parekh, S. Russ, D. A. Amsalem, N. Rambaran, and S. W. Wright, "Who leaves the emergency department without being seen? A public hospital experience in Georgetown, Guyana," $B M C$ Emergency Medicine, vol. 13, no. 1, 2013. [Online]. Available: 10.1186/1471-227x-13-10;https://dx.doi.org/10.1186/1471-227x-13-10

[9] J. Monzon, S. M. Friedman, C. Clarke, and T. Arenovich, "Patients who leave the emergency department without being seen by a physician: a control-matched study," CJEM, vol. 7, no. 02, pp. 107113, 2005. [Online]. Available: 10.1017/s1481803500013063;https: //dx.doi.org/10.1017/s1481803500013063

[10] M. Johnson, S. Myers, J. Wineholt, M. Pollack, and A. L. Kusmiesz, "Patients Who Leave the Emergency Department Without Being Seen," Journal of Emergency Nursing, vol. 35, no. 2, pp. 105108, 2009. [Online]. Available: 10.1016/j.jen.2008.05.006;https: //dx.doi.org/10.1016/j.jen.2008.05.006

[11] K. W. Arendt, A. T. Sadosty, A. L. Weaver, C. R. Brent, and E. T. Boie, "The left-without-being-seen patients: what would keep them from leaving?" Ann Emerg Med, vol. 42, pp. 317-323, 2003. 